\title{
Research on Control System of Ice Storage Air Conditioning Based on Fuzzy Control
}

\author{
Zhao Wei, Li Fangyun, Ge Jing \\ Institute of Technology. East China Jiaotong University, Nanchang Jiangxi, 330100, China
}

Keywords: fuzzy control; ice storage air conditioning; control system

\begin{abstract}
With the advanced development of the society, ice storage air conditioning system develops towards intellectualization. The control system of ice storage air conditioning is required to be equipped with parameters of intellectual regulation to control the temperature. In operation, ice storage air conditioning is with many interference factors, strong time-varying characteristics and many variables, thus, without fuzzy control, temperature control of control system of ice storage air conditioning may cannot satisfy people's needs. In order to improve the intellectualization of control system of ice storage air conditioning, a control system of ice storage air conditioning based on fuzzy control shall be established.
\end{abstract}

The refrigeration method of ice storage air conditioning is to apply expansion, evaporation, compression and cooling after determining the temperature control target to complete the cooling of the air in the space. The cooling control is that the chiller firstly makes the cold water of $4 \sim 10{ }^{\circ} \mathrm{C}$, and then sends the cold water to the cooler to complete the heat exchange. The ice storage air conditioning control system based on fuzzy control refers to the application of a set of fuzzy calculation and decision-making rules, so that the ice storage air conditioning control system can intelligently adjust the parameters and the heat exchange can reach the goal of temperature control.

\section{Basic Control Method of Ice Storage Air Conditioning Control System Refrigeration}

The parameters of ice storage air conditioning control system are adjusted to a temperature between 22 and $28{ }^{\circ} \mathrm{C}$, a relative humidity between $40 \%$ and $60 \%$, and a wind speed of no more than $0.3 \mathrm{~m} / \mathrm{s}$. To achieve such a goal, the parameters should be controlled according to the temperature difference heat transfer capacity and the fresh air load of the building envelope. The method of parameter controlling of ice storage air conditioning is described.

(1) The calculation method of cooling load

The calculation of ice storage air conditioning cooling load is shown in Equation (1).

$$
q_{e}=Q C_{q}
$$

In Equation (1), $Q$ is the heat dissipating capacity by the equipment machinery, and the unit is $W ; C_{q}$ is the cooling load coefficient of the equipment, and sometimes the air conditioning system is not continuously operated, then $C_{q}$ will be taken as 1.0 , otherwise it will be taken according to the actual cooling load coefficient.

Indoor lighting equipment will dissipate heat, and the cooling load of indoor lighting heat dissipation is described in Equation (2).

$$
q_{t}=1000 \mathrm{NC}_{q}
$$

In Equation (2), $N$ is the power of lighting equipment, and the unit is $W ; C_{q}$ is determined by lighting time.

People also dissipate heat, related to many factors. The cooling load caused by body heat dissipation is shown in Equation (3). 


$$
q_{p}=n \cdot g \cdot C_{q}
$$

In Equation (3), $n$ is the number of people; $g$ is the amount of heat dissipated for each person; $C_{q}$ is the sensible heat dissipation coefficient of the human body, which is determined by the time the person stays in the building.

(2) The calculation of air supply

The ice storage air conditioning requires the application of air supply to absorb the residual heat and residual moisture in the building, and control the temperature and humidity in the building. The calculation of the air supply is shown in Equation (4).

$$
L=\frac{Q}{\rho c \Delta t_{0}}
$$

In Equation (4), $L$ is the air supply, and the unit is $m^{3} / h$; $Q$ is the indoor total cooling load, and the unit is $\mathrm{kJ} / \mathrm{h} ; \rho$ is the air density, the unit is $\mathrm{kg} / \mathrm{m}^{3}$, and generally the value is $1.2 ; C$ is the air specific heat at constant pressure, the unit is $\mathrm{kJ} / \mathrm{kg} .{ }^{\circ} \mathrm{C}$, and the general value is $1.01 ; \Delta t_{0}$ is the temperature difference of the air supply, that is, the difference between the indoor temperature and the air supply temperature, and the unit is ${ }^{\circ} \mathrm{C}$.

(3) The establishment of parameter control model of space ice storage air conditioning

The space ice storage air conditioning parameter control model can be described as the rate of change of heat storage in the building $=$ the amount of heat entering the indoor air per hour + the heat of the indoor equipment lighting and the human body - the heat of the air discharged from the room per hour - the heat transfer out of the room from indoors per hour. The model is described in Equation (5).

$$
C \frac{d t_{n}}{d t}=L \rho c t_{s}+q_{n}-L \rho c_{n}-\frac{t_{n}-t_{0}}{R}
$$

In Equation (5), $t_{s}$ is the air supply temperature, $t_{n}$ is the indoor temperature, and the unit is ${ }^{\circ} \mathrm{C}$; $t_{0}$ is the temperature of the outdoor environment, and other parameters are shown in Equation (1)-(4).

An example is now applied to illustrate the method of model control. There is a space in which it has a volume of $5.4 \mathrm{~m} \times 3.3 \mathrm{~m} \times 3.25 \mathrm{~m}, t_{n}$ is known as $28^{\circ} \mathrm{C}, t_{s}$ is known as $18^{\circ} \mathrm{C}$, and $t_{0}$ is known as $35^{\circ} \mathrm{C}$. Parameters are set as $C=82.5 \mathrm{~s} 、 R=0.326 \mathrm{k} / \mathrm{kw} 、 \rho=1.2 \mathrm{~kg} / \mathrm{m}^{3} 、 C=1.01 \mathrm{~kJ} / \mathrm{kg} \cdot{ }^{\circ} \mathrm{C}$.. According to the model in Equation (5), the change of the model parameters can be adjusted by adjusting the air supply volume to achieve the air conditioning refrigeration.

\section{Fuzzy Control Principle and Controller Application Method of Ice Storage Air Conditioning Control System Refrigeration}

\subsection{Fuzzy control principle}

Set theory is the basis of the fuzzy control principle. Now suppose that there is an element $X$. Logically, it can be regarded as belonging to a set $A$ and not belonging to a set A. However, in real life, the element $X$ may have a fuzzy state, so people can only apply the feature function of element $X$ and set A to describe it, the function is shown in Equation (6).

$$
f_{A}(x)=\left\{\begin{array}{l}
1, x \in A \\
(0,1) x \in A \\
0, x \notin A
\end{array}\right.
$$

In Equation (6), the fuzzy state explores the extent to which the element $X$ belong to the set $\mathrm{A}$.

Now suppose that there is a finite field $A \times B$, then apply the principle of fuzzy sets and 
membership functions to explore it, shown in Equation (7).

$$
R=\int_{A \times B} \mu_{R}(x, y) /(x, y) \quad x \in A, y \in B
$$

The fuzzy matrix can be applied to complete the calculation of Equation (7), $A=\left\{x_{1}, x_{2}, \cdots, x_{n}\right\}$ and ${ }^{B}=\left\{y_{1}, y_{2}, \cdots, y_{m}\right\}$ are set to be finite set, and now the fuzzy relation $R$ is applied to describe the matrix $n \times m$ of $A \times B$, shown in Equation (8).

$$
R(A, B)=\left[\begin{array}{cccc}
\mu_{R}\left(x_{1}, y_{1}\right) & \mu_{R}\left(x_{1}, y_{2}\right) & \cdots & \mu_{R}\left(x_{1}, y_{m}\right) \\
\mu_{R}\left(x_{2}, y_{1}\right) & \mu_{R}\left(x_{2}, y_{2}\right) & \cdots & \mu_{R}\left(x_{1}, y_{m}\right) \\
\vdots & \vdots & & \vdots \\
\mu_{R}\left(x_{n}, y_{1}\right) & \mu_{R}\left(x_{n}, y_{1}\right) & \cdots & \mu_{R}\left(x_{n}, y_{m}\right)
\end{array}\right]
$$

The value of Equation (8) is in [0,1].

Now suppose that there is a direct product space $X \times Y=\{(x, y) \mid x \in X, y \in Y\}$, and the fuzzy relation existing in the space $X \times Y$ is a fuzzy subset $R$, and ${ }^{R(x, y)}$ can be applied to describe the degree of relationship between the fuzzy subset $R$ and the element $X$ in each set $X$ and the element $y$ in each set $Y$ in the direct product space. The calculation of $R(x, y)$ can apply the three calculation methods.

Intersect method is $R \cap S \leftrightarrow \mu_{R \cap S}(x, y)=\mu_{R}(x, y) \wedge \mu_{S}(x, y)$.

Union method is $R \cup S \leftrightarrow \mu_{R \cup S}(x, y)=\mu_{R}(x, y) \vee \mu_{S}(x, y)$.

Complementary operation method is $\bar{R} \leftrightarrow \mu_{\bar{R}}(x, y)=1-\mu_{R}(x, y)$.

If the logical inference rule of "if • then" is applied to describe "if $\times$ is A then $\mathrm{y}$ is B", then A and $B$ can be defined as the fuzzy linguistic variables of the universe of discourse $X$ and $Y$, and $A \rightarrow B$ can be applied to describe the logical relationship of $\mathrm{X} \times \mathrm{Y}$. Now four fuzzy calculation equations to calculate the fuzzy relationship under "if • then", which can be used as the basis for fuzzy control.

Fuzzy implication minimum calculation (Mandani) is Equation $R_{m}=A \rightarrow B=\int_{X \times Y} \mu_{A}(x) \wedge \mu_{B}(y) /(x, y)$

Fuzzy implication product calculation (Larsen) is Equation $R_{l}=A \rightarrow B=\int_{X \times Y} \mu_{A}(x) \mu_{B}(y) /(x, y)$

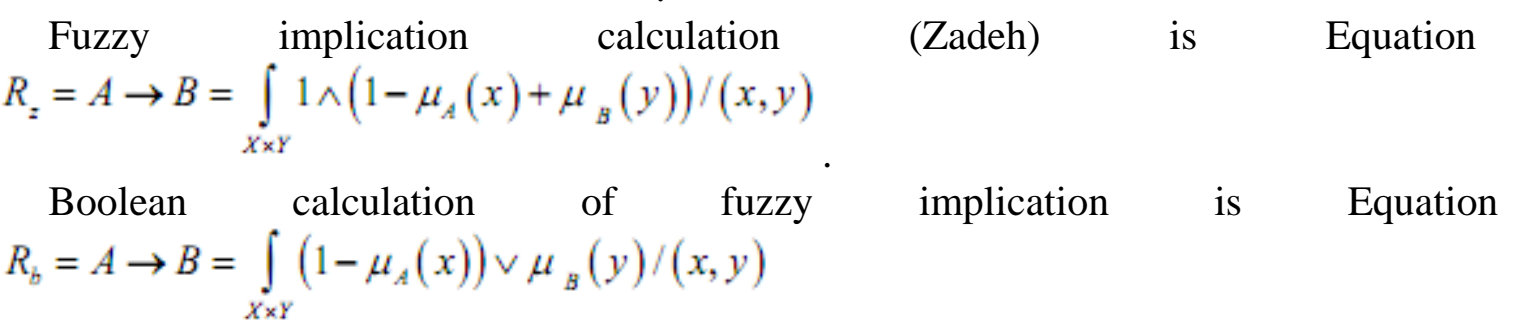

In fuzzy control, the equation can be selected according to the requirements of control, which is the key for intelligent control of computer.

\subsection{The establishment of fuzzy control system}

The fuzzy control system refers to the system that applies the principle of fuzzy control according to the requirements, allows the computer to intelligently perform fuzzy calculation, and then gives the control scheme. The fuzzy control system can be used to establish a fuzzy control scheme based on the requirements of ice storage air conditioning control. The ice storage air conditioning fuzzy control system is shown in Figure 1. 


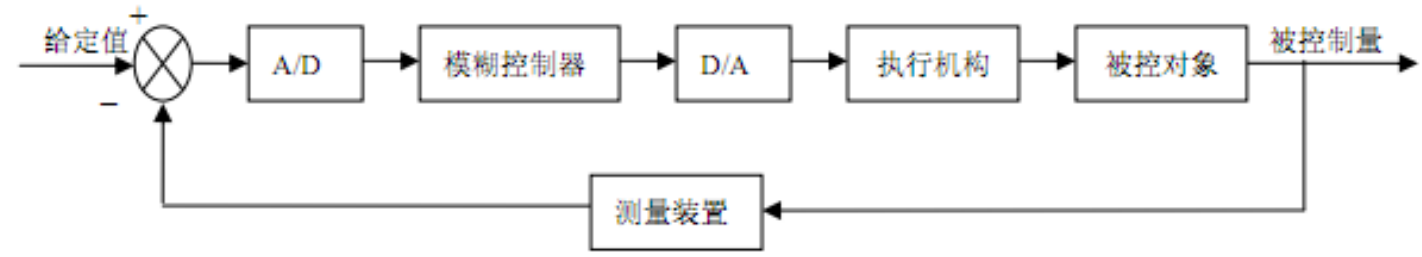

Figure 1 The ice storage air conditioning fuzzy control system

In this system, the set value is the target of the control. The controlled variable refers to the numerical parameter after the fuzzy control. After the controlled variable parameter is obtained, the system compares it with the set value. The control is completed if reaching the set value; otherwise, the intelligent adjustment parameter must continue until the controlled amount reaches the set value. The controlled object refers to the controlled device or equipment group, and now the ice storage air conditioning. The actuator refers to the device construction in the device that can adjust the parameters. The fuzzy controller is a hardware device for fuzzy calculation. In the ice storage air conditioning control system, the hardware device that can be used as the fuzzy controller include computer, a PLC, single chip microcomputer, industrial control machine, and the like. The fuzzy controller is responsible for completing the entire calculation process of the fuzzy control, including the process of data collection, calculation, and the process of giving control decision scheme. A/D or $\mathrm{D} / \mathrm{A}$, is the input interface and output interface of the fuzzy controller, responsible for receiving the instructions of the fuzzy controller and completing the control operation. Measuring equipment is the device for collecting and processing data.

\subsection{Application method of fuzzy controller}

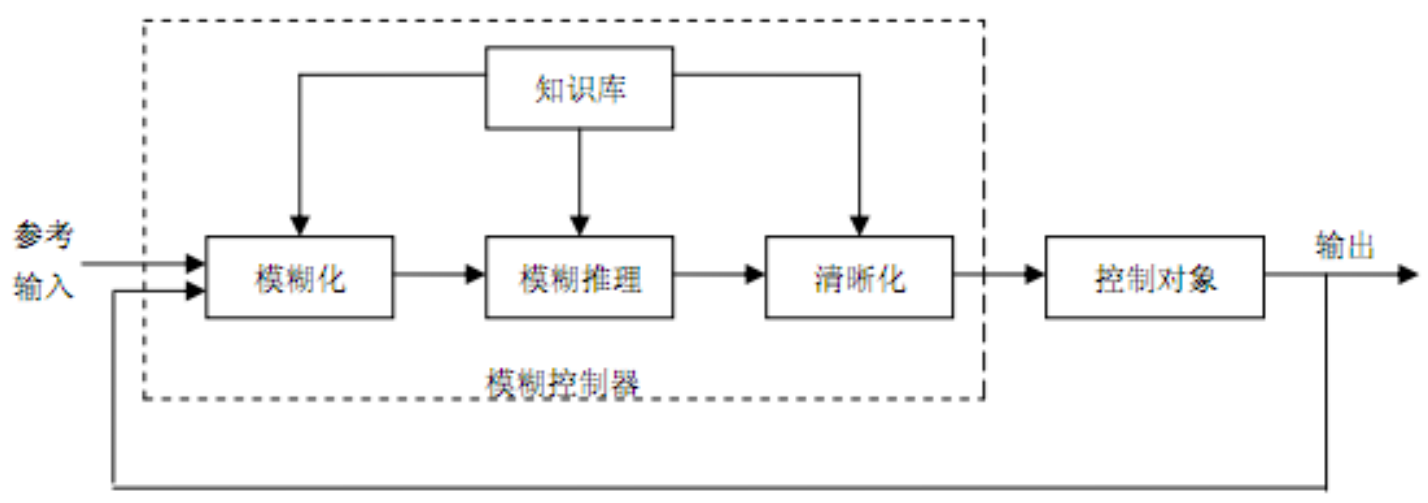

Figure 2 The structure of fuzzy controller

The structure of fuzzy controller is shown in Figure 2. It applies A/D or D/A to control input and output. The application goal of fuzzy controller is to apply fuzzy controller to complete intelligent fuzzy judgment. The application procedure is described in detail. First, intelligent fuzzy controller is applied to judge the goal and the result, the intelligent controller can take the input as the control goal, take controlled object as control carrier, and collect the data after the control command is issued, and analyze the gap between control result and control goal to determine the subsequent control operations until the control goal is reached. The process of fuzzification controller is to analyze the target parameter and change it into the input required by the fuzzy control parameter, and $\mathrm{y}$ is applied to represent the output parameter, E to represent the error, and EC to represent the error change rate; the input is included in the universe of discourse for the subsequent processing; the calculation equation set by the fuzzy controller is used for calculation, the parameter result is obtained, and the result of the parameter control is taken as the control scheme. Second, the data and rules in the knowledge base are applied to provide material and basis for the calculation of the intelligent controller. The knowledge base contains data information base and rule information base. The data information base contains input and output variables, all of which belong to the vector values in the fuzzy subset; the rule information base contains a large number of functions. When applying the fuzzy calculation equation for data calculation, functions in rule information base need 
to be applied to complete the calculations. For example, the rule information base includes calculation rules such as "if-then", "else", and "also". Third, the rules in the rule base are applied to collect data and complete the fuzzy calculation. The rule inference calculation in the fuzzy controller simulates human thinking, and it can analyze the results of data calculation by fuzzy judgment. Fourth, the results of the fuzzy calculation are clarified. Although the answer obtained by fuzzy calculation is a set of unclear answers, the instructions issued by the fuzzy controller must be clear. The fuzzy controller must clarify the results of the fuzzy calculations and give a data parameter that can be clearly executed. The method of clarifying the fuzzy data is to judge the range of the numerical value, and give the corresponding decision judgment according to the range.

\section{Simulation Example of Ice Storage Air Conditioning Control System Based on Fuzzy Control}

In order to illustrate the application method of ice storage air conditioning control system based on fuzzy control, a simulation example is applied to illustrate how to apply fuzzy controller to intelligently control ice storage air conditioning refrigeration.

(1) The use of fuzzy toolbox

The MATLAB fuzzy toolbox is used as a simulation tool, which can completely simulate the working process of the fuzzy controller. The application advantage of this tool is that it provides a graphical interface with good interface affinity, which enables people to intuitively understand how to complete the input operation, and can graphically present the results of the simulation; it provides a fuzzy reasoning system editor, the membership function editor, the fuzzy rule function editor, the fuzzy rule observer, the curve of fuzzy reasoning output, so that the function of fuzzy control can be fully simulated; the dynamic connection is available, and a fuzzy control system can be established according to the requirements.

(2) The realization of fuzzy control

Step 1. Temperature is set as control goal.

Set the temperature error as the E parameter, and the change of the temperature error is set to the EC parameter and the air supply amount should be expressed by U. Open the MATLAB fuzzy toolbox for the FIS Editor tool and open the Edit/add input menu to create these three variables. Set the calculation method to "intersection", "union", "complement", and now choose Mandani as the control operation method.

Step 2. To set scaling factor and correlate E, EC, and U parameter with $k_{e} 、 k_{e c} 、 k_{u}$. The scaling factor affects the update frequency of the parameter. If the scaling factor is set too large, the calculation load will be large, and sometimes there will be a steady-state error; if the setting is too small, the parameter update frequency will be too slow, then the speed of parameter update may cannot keep up with the need for control adjustment, so there is a large error in the control goal and output. For the convenience of calculation, the universe of discourse of temperature error is now set to $[-5,5]$, set to 1.2 ; now the temperature error is set to $[-10,10]$, set to 0.6 ; the range of $k_{u}$ is set to $[65,550]$ and set to 0.001 . The simulation range of the MATLAB fuzzy toolbox is [-550, +550], and the set value is within the preset range to meet the needs of the toolbox calculation.

Step 3. To establish membership function. The purpose of establishing a membership function is to establish a control level. If too many membership functions are established, although the actuarial control will be higher, the calculation process will be more complicated, which may bring a large load to the fuzzy controller calculation; and if the calculation process cannot keep up with the parameter value update, the process then affects the fluency of the control. Now five levels $\{N B$ NS ZR PS $P B\}$ are established. Set the function level corresponding to E and EC to the value range $[-6,6]$, and set the probability to $[0,1]$. Set the curve of membership function of E, EC and output $U$, and the corresponding value of the function level should be set in the range of $[-6,6]$, and the degree of possibility is $[0,1]$.

Step 4. To establish fuzzy control rule. The establishment of fuzzy control fuzzy of ice storage 
air conditioning control system is shown in Table 1 . The weighted default of each rule is set as 1 . From Table 1, there are 25 control rules in the control system.

Table 1 Fuzzy control fuzzy of ice storage air conditioning control system

\begin{tabular}{|l|l|l|l|l|l|}
\hline & NB & NS & ZR & PS & PB \\
\hline NB & NB & NB & NS & NS & ZR \\
\hline NS & NB & NS & NS & ZR & ZR \\
\hline ZR & NS & NS & ZR & ZR & PS \\
\hline PS & NS & ZR & ZR & PS & PS \\
\hline PB & ZR & ZR & PS & PS & PB \\
\hline
\end{tabular}

Step 5. To establish fuzzy control simulation system. Now assume that in a space, the fuzzy control simulation system is applied to complete the control of the ice storage air conditioning, then the system can be described in Figure 3.

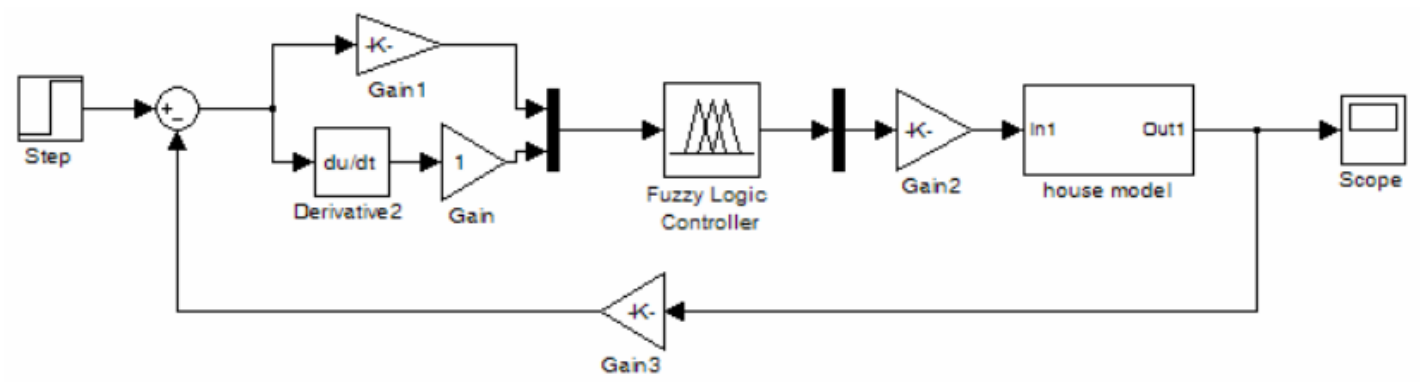

Figure 3 The simulation system of ice storage air conditioning based on fuzzy control

(3) The presentation of fuzzy control result

The above method is used to establish the ice storage air conditioning control simulation system based on fuzzy control. The air conditioning temperature is 25 degrees in the simulation system. The control result of the simulation system is shown in Fig. 4. From Figure 4, the boundary is 600s. The temperature fluctuated up and down in the dynamic control parameters of the control system before 600 s, and after 600 s, the temperature is constant in the control system.

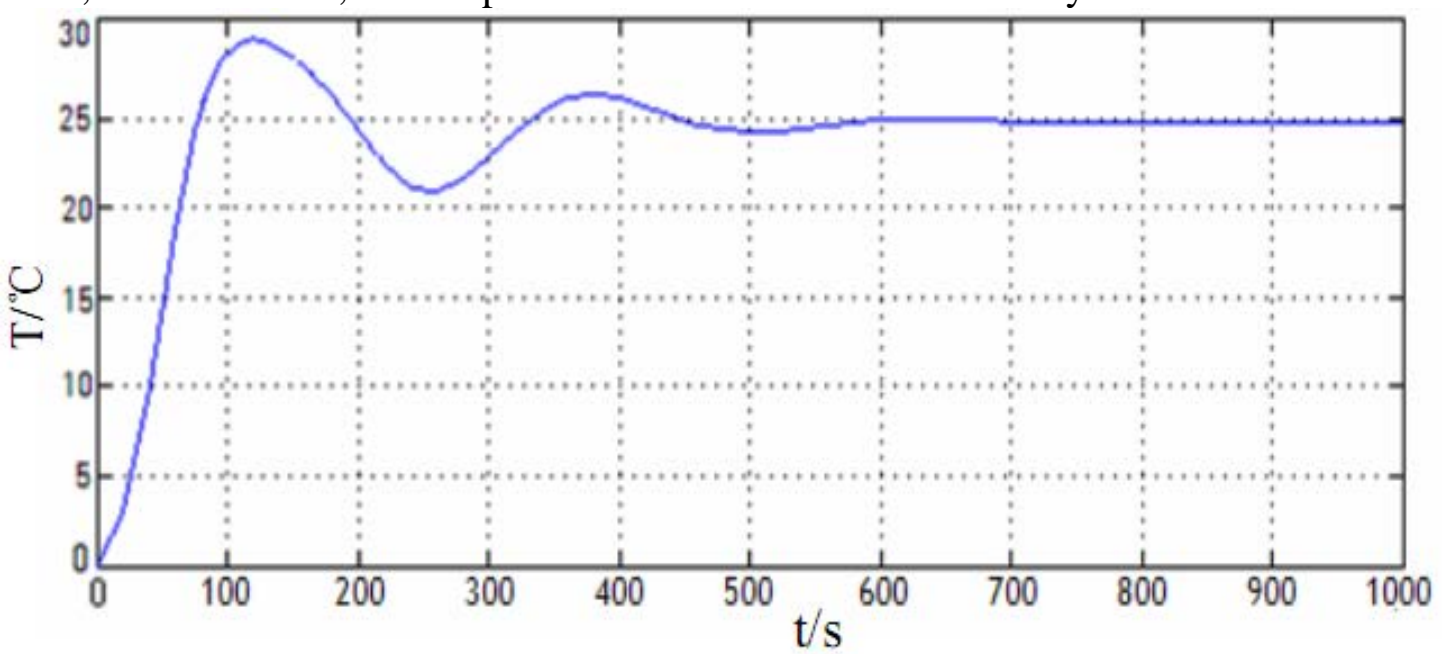

Figure 4. The output result of ice storage air conditioning simulation system (1)

The temperature error is now set to $[-4,4]$, and the remaining parameters are unchanged. Figure 5 shows the output of the ice storage air conditioning control simulation system. From Figure 5 , the boundary is still 600s, the temperature fluctuated up and down in the temperature control before 600 s, but the amplitude of the fluctuation is small. 


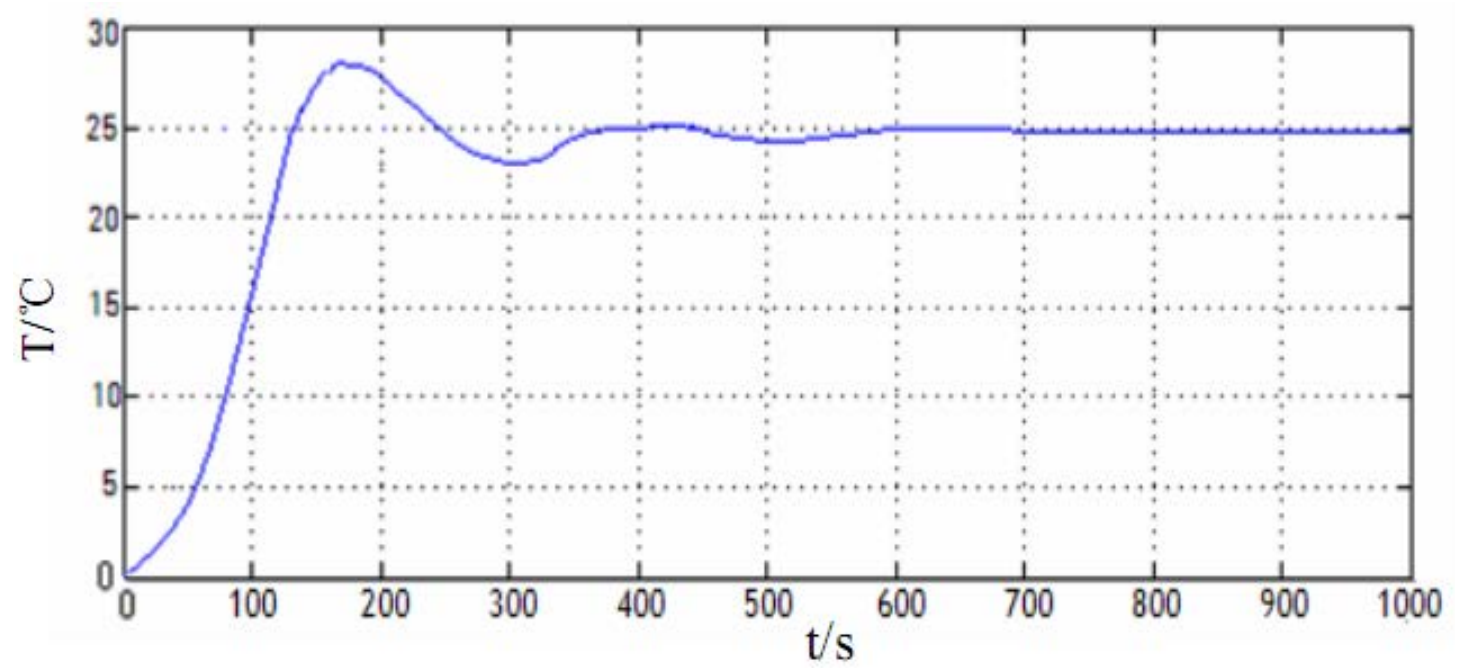

Figure 5 The output result of ice storage air conditioning simulation system (2)

Now the temperature error is set to $[-4,4]$, the five control levels are set to 7 levels, and the remaining parameters are unchanged. Figure 6 shows the output of the ice storage air conditioning control simulation system. From Figure 6, the boundary is still 600s, the temperature fluctuated up and down in the temperature control before 600s, but the fluctuation is smooth.

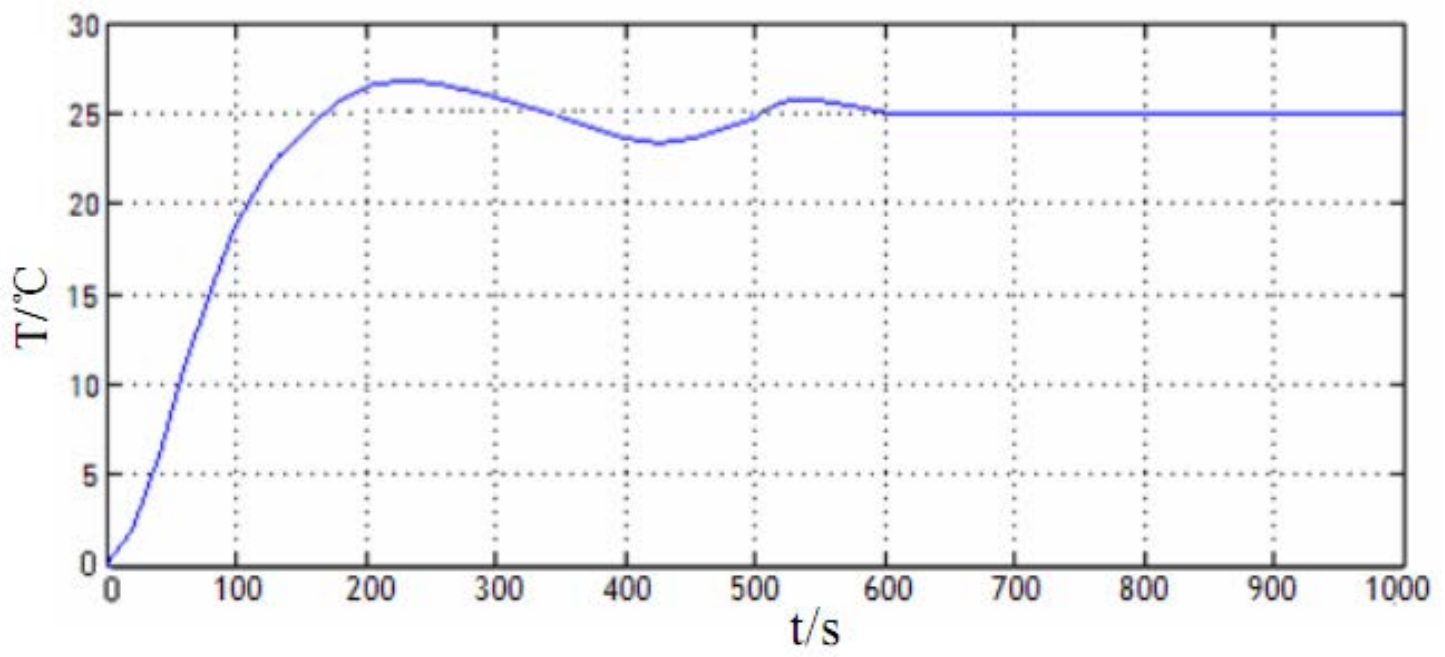

Figure 6 The output result of ice storage air conditioning simulation system (3)

\section{Conclusion}

The ice storage air conditioning control system based on fuzzy control is based on the ice storage air conditioning structure, with constant temperature in the space as the goal, and the air conditioning refrigeration results are controlled by temperature error, temperature error change and air supply volume. The system uses the fuzzy controller as the hardware carrier, and the intelligent fuzzy computing software module in the fuzzy controller as the core to complete intelligent adjustment and control of the temperature. Application simulation examples show that in the ice storage air conditioning control system, the fuzzy control system can be applied, and the intelligent control of ice storage air conditioning control system can be completed by optimizing the setting range of the three control parameters. The ice storage air conditioning control system with fuzzy control can make the ice storage air conditioning intelligently complete the cooling regulation and reduce the cost of temperature control.

\section{Acknowledgement}

Fund project: This paper comes from scientific and technological research project of Jiangxi 
Province Department of Education. Project Name: Research on Control System of Ice Storage Air Conditioning Based on Fuzzy Control. Project No. 161560.

\section{References}

[1] Qi Yebai, Wang Dan, Jia Hongjie, Huang Renle, Zhang Yi, Yang Zhanyong. Demand Response Control Strategy for Central Air-conditioner Based on Temperature Adjustment of Partial Terminal Devices [J]. Automation of Electric Power Systems, 2015(17).

[2] Sui Xuemin, Zhang Xu, Han Guanghui. Select of Indoor Thermal Environment Control Variables in Radiant Cooled Room [J]. Fluid Machinery, 2013(03).

[3] Xu Yong. Energy Consumption Analysis and Energy-conservation Control of Air Conditioning with Constant Temperature and Humidity [J]. Architectural Design Management, 2010(04). 